\title{
El método de Monte Carlo y los desarrollos asintóticos
}

\author{
The Monte Carlo method and the asymptotic
}

\author{
Fanny Campomanes Murrugarra ${ }^{1}$
}

\begin{abstract}
Resumen
En el presente trabajo recordamos con algún detalle el Método de Monte Carlo para su aplicación y contraste posteriores. Después de mencionar las distribuciones de Pearson, se va a examinar las condiciones para hallar desarrollos asintóticos que las aproximen; a continuación, se proponen los desarrollos asintóticos para la distribución Beta. También se presenta un cálculo aproximado de una integral propia de la distribución Beta utilizando el método de Monte Carlo, concluyendo que dicho método es más rápido que otros de aproximación.
\end{abstract}

Palabras clave: desarrollos asintóticos; distribución de Beta; distribuciones de Pearson; método de Monte Carlo.

\begin{abstract}
In the present work we recall in some detail The Monte Carlo Method for later application and contrast. After mentioning Pearson's distributions, the conditions will be examined to find asymptotic development that approximate them; then the asymptotic development for the beta distribution are proposed. An approximate estimate of an integral of the Beta distribution is also presented using the Monte Carlo method, concluding that the Monte Carlo method is faster than other approach method.
\end{abstract}

Keywords: asymptotic expansions; Beta distribution; Pearson distributions; Monte Carlo method.

\section{Introducción}

En el presente trabajo se recurre al método de Monte Carlo, el que ha sido usado por los investigadores para dar salida a problemas matemáticos con los que se encuentran en la teoría de probabilidades y en la estadística matemática. El método de Monte Carlo ha sido reemplazado, a lo largo del tiempo, por otros como, por ejemplo, el de las aproximaciones asintóticas.

Se presenta un punto de vista sincrético mencionando, en forma general, a las distribuciones de Pearson que, como es sabido, abarca un amplio número de distribuciones de la teoría de probabilidades (beta, gamma, normal, etc.)

El método de Monte Carlo se puede usar para analizar sistemas de servicios, análisis de la calidad y seguridad de piezas, análisis de paso de neutrones a través de una placa y para un numeroso conjunto de problemas propios de la medicina.

Otra de las aplicaciones del método Monte Carlo es el cálculo aproximado de las integrales definidas, tema elegido en el presente trabajo, y que se presenta acompañado de algunos detalles. Hay métodos numéricos que permiten mejores aproximaciones al problema planteado, pero el grado de aproximación que se exige a un método determinado es, en última instancia, una decisión ingenieril. Los aspectos matemáticos no son afectados por la prevalencia de uno u otro método en los procesos de cálculo, los cuales son influenciados por el uso. Además, el método Monte Carlo nos proporciona una solución rápida, aunque no extremadamente exacta del problema planteado.

\section{Materiales y métodos}

\section{El método Monte Carlo}

Este es un método numérico que permite resolver problemas matemáticos mediante la simulación de variables aleatorias.

\section{Orígenes}

El año 1949 se considera como el del nacimiento del método Monte Carlo, debido a la aparición del artículo "The Monte Carlo method". Su creación suele vincularse a los nombres de los matemáticos norteamericanos J. Von Neumann y S. Ulam; sin embargo, la base teórica del método se conocía desde mucho antes. Se puede afirmar que algunos problemas de la estadística se resolvían empleando las muestras aleatorias, es decir, aplicando, de hecho, el método Monte Carlo.

La aparición de las computadoras digitales y la difusión de su uso hicieron posible ampliar las aplicaciones,

\footnotetext{
Jefe de práctica, Departamento de Estadística e Informática. Universidad Nacional Agraria La Molina, Lima, Perú. Email: fcampomanes@lamolina.edu.pe 
pues la simulación a mano de variables aleatorias era y es un proceso engorroso. Las máquinas calculadoras electrónicas hicieron del Monte Carlo un método numérico universal.

\section{El método}

El método Monte Carlo tiene dos peculiaridades. La primera consiste en que su algoritmo tiene una estructura muy sencilla. Como regla, se elabora primero un programa para la elaboración de una prueba aleatoria, después, esta prueba se repite un número determinado de veces, de modo que cada experimento sea independiente de los restantes, y se toma la media de los resultados de todos los experimentos. Por esto, el método Monte Carlo se llama también el método de pruebas estadísticas.

La segunda peculiaridad consiste en que el error es, como regla, proporcional a la magnitud $\sqrt{ }(\mathrm{D} / \mathrm{N})$ donde es una constante y el número de pruebas. Esta fórmula permite ver que para disminuir el error en 10 veces es necesario aumentar en 100 veces. Es necesario advertir la imposibilidad de alcanzar en este camino una elevada exactitud. Suele decirse que el método Monte Carlo resulta especialmente eficaz en la solución de problemas en los cuales se necesita conocer el resultado con poca exactitud (del 5 al 10\%).

¿Qué problemas se pueden resolver por el método Monte Carlo?

Este método permite simular cualquier proceso cuya marcha depende de factores aleatorios. También es posible, en muchos problemas matemáticos, que no tienen la menor relación con procesos aleatorios, inventar un modelo probabilístico artificial (o más de uno) que permita resolver estos problemas. Ejemplo típico de lo segundo es el cálculo de áreas y superficies.

\section{Esquema general de aplicación del método Monte Carlo}

Es conocido que, a partir del Teorema de Chebyshev y la Ley de los Grandes Números, podemos afirmar lo siguiente:

\section{Teorema}

Si la sucesión de magnitudes aleatorias independientes $\left\{\mathrm{x}_{\mathrm{n}}, \mathrm{n} \geq 1\right\}$ es tal que la varianza $\mathrm{V}\left(\mathrm{x}_{\mathrm{n}}\right)$ existe y $\left(\mathrm{V}\left(\mathrm{x} \_\mathrm{n}\right)\right) /$ $\mathrm{n} \rightarrow 0$ cuando $\mathrm{n} \rightarrow \infty$ entonces

$\frac{1}{n} \sum_{k=1}^{n} x_{k}-\frac{1}{n} \sum_{k=1}^{n} E\left(x_{k}\right) \rightarrow 0$

es decir, converge a 0 en probabilidad.

\section{Teorema}

Si $\left\{\mathrm{x}_{\mathrm{n}}, \mathrm{n} \geq 1\right\}$ es una sucesión de magnitudes aleatorias independientes igualmente distribuidas y si existe la esperanza matemática $\mathrm{E}\left(\mathrm{x}_{\mathrm{k}}\right)=\mathrm{m}$, entonces, para $\mathrm{n} \rightarrow \infty$

$$
\frac{1}{n} \sum_{k=1}^{n} x_{k} \rightarrow m
$$

Sea $\mathrm{x}$ una variable aleatoria normal $\mathrm{N}(\mu, \sigma)$

La densidad es: $p(t)=\frac{1}{\sqrt{2 \pi} \sigma} e^{-\frac{(t-\mu)^{2}}{2 \sigma^{2}}}$

Se calcula $\quad \int_{\mu-3 \sigma}^{\mu+3 \sigma} p(t) d t=0.997$
Cualesquiera que sean $\mu$ y $\sigma$

En consecuencia, se puede afirmar:

$\mathrm{P}\{\mu-3 \sigma<\mathrm{x}<\mu+3 \sigma\}=0.997$

Lo que habitualmente se interpreta así:

"Al efectuar una prueba es prácticamente imposible obtener un valor de $x$ que difiera de $E(x)$ en más de $3 \sigma$ ".

Ahora, calculando una magnitud $r$ que se desconoce. Sea una variable aleatoria $x$ tal que $E(x)=r$. Además, $V(x)=b^{2}$ Se considera $N$ variables aleatorias independientes $x_{1}, x_{2}, x_{3}, \ldots, x_{N}$ con la misma distribución que tiene $x$. Si $N$ es suficientemente grande, la distribución de la suma $\sum_{k=1}^{N} x_{k}$ será una distribución normal con parámetros $\mu=$ $N r y \sigma^{2}=N b^{2}$.

De: $\mathbf{P}\{\mu-3 \sigma<x<\mu+3 \sigma\}=0,997$

Se deduce: $\mathbf{P}\left\{N r-3 b \sqrt{N}<\sum_{k=1}^{N} x_{k}<N r+3 b \sqrt{N}\right\} \approx 0,997$

$\mathrm{O}$ lo que es equivalente

$$
\mathbf{P}\left\{r-\frac{3 b}{\sqrt{N}}<\sum_{k=1}^{N} x_{k}<r+\frac{3 b}{\sqrt{N}}\right\} \approx 0,997
$$

Se representa

$$
\mathbf{P}\left\{\left|\frac{1}{N} \sum_{k=1}^{N} x_{k}-r\right|<\frac{3 b}{\sqrt{N}}\right\} \approx 0,997
$$

Relación que es de suma importancia para el método Monte Carlo ya que ofrece un método para calcular $r$ y a la vez permite estimar el error. Al determinar $N$ valores de la variable aleatoria, la media aritmética de estos será aproximadamente igual a $r$. Con una alta probabilidad, se puede afirmar que el error no pasa de $3 b / \sqrt{ } N$. El error tiende a cero cuando $N$ crece.

\section{LAS DISTRIBUCIONES DE PEARSON}

Se llaman de Pearson las distribuciones continuas cuyas densidades de probabilidad son las soluciones de la ecuación diferencial

$$
\frac{d f}{d x}=\frac{a_{1} x+a_{0}}{b_{0}+2 b_{1} x+b_{2} x^{2}} \cdot f(x)
$$

Donde $\mathrm{a}_{0}, \mathrm{a}_{1}, \mathrm{~b}_{0}, \mathrm{~b}_{1}, \mathrm{~b}_{2}$ son parámetros de la distribución. Las raíces del trinomio cuadrado $b_{0}+2 b_{1} x+b_{2} x^{2}$ nos sirven para indicar los tipos de las distribuciones de Pearson, que son 12.

Desarrollos asintóticos de las distribuciones de

\section{Pearson}

\section{Consideraciones generales}

Sea $\mathrm{f}(x, t)$ la función de densidad y $\mathrm{F}(x, t)$ la función de distribución de alguna distribución continua que depende del parámetro $t$ (en nuestro caso y para lo que sigue, se asume que $f$ y $F$ están definidas para todo $t \in \mathfrak{I}$, y que $\mathrm{t}=0$ es un punto de acumulación del conjunto $\mathfrak{I}$ ).

Cuando $t \rightarrow 0 \mathrm{~F}(x, t)$ aproxima la función de distribución $\Phi(x)$ de alguna distribución continua pero la convergencia puede no ser satisfactoria, la fórmula de aproximación $\mathrm{F}(x, t) \approx \Phi(x)$ puede volverse irrealizable en la práctica $\mathrm{y}$ 
requerir refinamientos. En cuyo caso, $F$ es usualmente reemplazada por una expresión asintótica que es una suma parcial obtenida al expandir $F$ en términos de algunas funciones ortogonales.

La dificultad básica asociada a cada "refinamiento polinómico" de la aproximación $\mathrm{F} \approx \Phi$ está vinculada a la estimación del error cuando $t \rightarrow 0$. Si ahora se llama "función de peso" a la función $\varphi(x)=\Phi$ ' $(x)$, para la que se asume como regla general que la razón $((\mathrm{F}-\Phi)) / \varphi, \mathrm{y}$ no $F$, es expandible en series. La función $\varphi(x)$ debe ser tal que la expansión en series de $F$ sea idéntica a la integral de la expansión en series de $f$. Se asume siempre que la densidad limitante es "función de peso" del sistema de polinomios ortogonales (Montel, 1928).

En otras palabras, se requiere que la expansión sea invariante con respecto a las operaciones de diferenciación e integración, lo que impone fuertes restricciones a la "función de peso" $\varphi$, las que se pasa a indicar.

Sea la densidad $\varphi(x)$ positiva y diferenciable en el intervalo $\mathrm{A}<x<\mathrm{B}$ e igual a cero fuera del mismo.

Para garantizar la invariancia antes mencionada se requiere, para todo polinomio $U(x)$ que satisfaciendo la condición

$\mathbf{M}|U|=\int_{A}^{B}|U(x)| \varphi(x) d x<\infty$

que las representaciones

(i) $\int_{A}^{x} U(x) \varphi(x) d x \equiv M \Phi(x)+\varphi(x) V(x)$

(ii) $U(x) \varphi^{\prime(x)} \equiv(L x+N) \varphi^{\prime}(x)+\varphi(x) W(x)$

encierran, donde $V y W$ son polinomios y L, My $N$ son constantes dependientes de la elección de $U$; se asume que $M=\mathrm{M} U$.

La condición (i) significa que, si la esperanza matemática del polinomio es igual a 0 , entonces la operación de integración no modifica la forma de la expansión. La condición (ii) emerge debido a que, en lo sucesivo, la operación $(\varphi U)^{\prime}$ 'se aplicará a los polinomios $U$ para los cuales $\varphi \mathrm{U} \rightarrow 0$ si $x \rightarrow \mathrm{A}$ o $x \rightarrow \mathrm{B}$, y en el caso simple de un intervalo finito $(A, B)$, usualmente se tiene $\mathrm{U}(A)=$ $\mathrm{U}(B)=0$.

En consecuencia, para cada polinomio sería natural escribir la condición de invariancia como $\mathrm{U} \varphi{ }^{\prime} \equiv \varphi \mathrm{W}$

En el caso más general, podemos darle a esta condición la forma $\mathrm{U} \varphi^{\prime} \equiv \mathrm{T} \varphi \varphi^{\prime}+\varphi W$

Donde $T$ es un polinomio de grado determinado, y además si $U(A)=U(B)=0$, entonces $T \equiv 0$

Ahora, para hallar alguna densidad que satisfaga las condiciones planteadas, se hace:

$\mathrm{U}=x$ en (i). Después de diferenciar, se obtiene $x=M+$ $V^{\prime}(x)+V(x)\left(\varphi^{\prime} / \varphi\right)$

Identidad que sólo es válida si

(iii)

$$
\frac{\varphi^{\prime}}{\varphi}=\frac{\omega(x)}{\Omega(x)}
$$

Esto es $x-M-V^{\prime} \equiv \omega v \quad$ y $\quad V \equiv \Omega v$

$\omega, \Omega$ y $v$ son polinomios.
Los polinomios $\omega$ y $\Omega$ son primos entre sí. Si definimos $S[\mathrm{U}]$ al grado de un polinomio arbitrario $U$. Si $\mathrm{S}[\omega] \geq$ $S[\Omega]$, entonces $1=\mathrm{S}[\omega]+\mathrm{S}[v]$ por la condición (iii) $\mathrm{y}$, en consecuencia $\mathrm{S}[\omega] \leq 1$. Pero si $S[\omega]+1<S[\Omega]$, entonces se halla que $S[\Omega]=2$. En vista de que la condición (ii) es satisfecha independientemente de para y $U=0,1$ y $x, y$ $\mathrm{U}=\mathrm{x}^{2}$ en (ii), se obtiene:

$$
\frac{\varphi^{\prime}}{\varphi}=\frac{\omega}{\Omega}=\frac{W(x)}{x^{2}-L x-N}
$$

Se sabe que $S[\omega]+1=S[\Omega]$, entonces $S[\mathrm{~W}] \leq 1$, lo que significa que $\mathrm{S}[\omega] \leq 1$.

Se probó lo siguiente:

\section{Teorema}

Para satisfacer conjuntamente las condiciones de convergencia de (i) y (ii), es necesario en el intervalo $\mathrm{A}<x<\mathrm{B}$ que la densidad $\varphi(x)$ satisfaga la ecuación diferencial de la forma

$$
\frac{\varphi^{\prime}}{\varphi}=-c_{0}(x) \equiv \frac{\omega(x)}{\Omega(x)} \equiv \frac{a_{1} x+a_{0}}{b_{2} x^{2}+2 b_{1} x+b_{0}}
$$

Donde $\mathrm{a}_{\mathrm{i}} \mathrm{y} \mathrm{b}_{\mathrm{j}}$ son coeficientes constantes.

Ecuación diferencial que define las distribuciones de Pearson. Un estudio de las mismas muestra que sistemas completos de polinomios ortogonales de todos los grados existen sólo para funciones $\varphi$ que sean densidades de la normal, distribución $\Gamma$ y distribución $B$.

Es necesario señalar que sólo hay tres diferentes tipos de sistemas de polinomios ortogonales completos que satisfacen las condiciones (i) y (ii), y son los polinomios de Chebyshev-Hermite, los polinomios de Laguerre y los polinomios de Jacobi (los polinomios de Legendre y los de Chebyshev son casos especiales de los polinomios de Jacobi).

\section{Ejemplo}

Considerando la densidad

$$
\varphi=\frac{m+1}{a}(1+x / a)^{m}
$$

Que, efectivamente es una distribución de Pearson en la que: $-\mathrm{a}<x<0 \mathrm{y}-1<\mathrm{m} \leq$

En este caso

$$
\Phi=(x+a) \varphi(x) / m+1
$$

Se tiene

$\int_{-a}^{x}(x+a)^{n} \varphi(x) d x=\frac{m+1}{m+n+1} a^{n} \Phi+\varphi V$

Donde $\mathrm{V} \rightarrow 0$ si $x \rightarrow 0$

\section{Expansión asintótica de la función de distribución}

Se asume que la distribución de probabilidades con densidad $f(x, t)$ satisface las condiciones siguientes:

I. La derivada logarítmica $\mathrm{f}^{\prime} / \mathrm{f}=-\mathrm{c}(\mathrm{x}, \mathrm{t})$ existe en el intervalo $\mathrm{A}<x<\mathrm{B}$ para todo $t \in \mathfrak{T}$. Se puede hallar, dentro del intervalo (A,B), para cada $t \in \mathfrak{T}$ un segmento $\mathrm{a}_{\mathrm{t}} \leq x \leq \mathrm{b}_{\mathrm{t}}$ en el cual la expansión

$$
c(x, t)=\sum_{k=0}^{m} c_{k}(x) t^{k}+t^{m+1} R_{m}(x, t)
$$


converge; donde $m$ es un entero no negativo $c_{1}(x) \ldots c_{m}(x)$ son polinomios que dependen sólo de $x \mathrm{y} \mathrm{c}_{0}=\varphi^{\prime} / \varphi$. Para todo $t \in \mathfrak{I}$ el resto $\mathrm{R}_{m}$ en el segmento $\mathrm{a}_{t} \leq x \leq \mathrm{b}_{t}$ satisface la desigualdad

$\left|\mathrm{R}_{m}(x, t)\right| \leq \mathrm{C}_{1}\left(1+|\mathrm{x}|^{\mathrm{m} 1}\right)$

Donde $\mathrm{m}_{1}=\mathrm{m}_{1}(\mathrm{~m})>0$ y $\mathrm{C}_{1}$ es una constante positiva, $\tan$ grande como se requiera.

II. En el segmento $\mathrm{a}_{\mathrm{t}} \leq x \leq \mathrm{b}_{\mathrm{t}}$ la razón $\varphi / f$ está uniformemente limitada para todo. Esto es $\varphi(x) \leq \mathrm{C}_{2} f(x, t)$ donde $\mathrm{C} 2 \mathrm{~s}$ es una constante absoluta.

III. La distribución con densidad $f(x, t)$ tiene momento de orden $\mathrm{m}_{2}>0$ para todo $t \in \mathfrak{I} \mathrm{y}$, además

$$
\left|\int_{-\infty}^{\infty} x^{k} f(x, t) d x\right| \leq C_{3}
$$

Donde $0 \leq \mathrm{k} \leq \mathrm{m}_{2} \mathrm{y} \mathrm{C}_{3}$ y es una contante absoluta.

\section{Lema}

Si las condiciones (i), (ii) y I son satisfechas, entonces las soluciones $\mathrm{H}_{1}(x), \ldots, \mathrm{H} m(x)$ de las ecuaciones diferenciales

$(\varphi \Omega H r)^{\prime \prime}+\mathrm{c}_{0}\left(\varphi \Omega H_{r}\right)^{\prime}+\mathrm{c}_{\mathrm{r}} \varphi=0 \quad$ si $r=1$
$\left(\varphi \Omega H_{r}\right)^{\prime \prime}+c_{0}\left(\varphi \Omega H_{r}\right)^{\prime}+c_{r} \varphi=-\sum_{k=1}^{r-1} c_{r-k}\left(\varphi \Omega H_{k}\right)^{\prime} \quad$ si $r=2, \ldots, m$ con las condiciones iniciales

$\lim _{x \rightarrow A} \varphi \Omega H_{r}=\lim _{x \rightarrow B} \varphi \Omega H_{r}=0 \quad r=1, \ldots, m$

son polinomios.

IV. Si $t \rightarrow 0$, entonces

$F\left(\mathrm{~b}_{\mathrm{t}}, \mathrm{t}\right)-\mathrm{F}\left(\mathrm{a}_{\mathrm{t}}, t\right)=1+O\left(\mathrm{t}^{\mathrm{m}+1}\right)$ y $\Phi\left(b_{t}\right)-\Phi\left(\mathrm{a}_{\mathrm{t}}\right)=1+O\left(\mathrm{t}^{\mathrm{m}+1}\right)$

Además, en las semirrectas $\quad \mathrm{x} \leq \mathrm{a}_{\mathrm{t}} \quad \mathrm{y} \quad \mathrm{x} \geq \mathrm{b}_{\mathrm{t}}$ las relaciones $\varphi(x) \Omega(x) \mathrm{H} r(x)=O\left(t^{m+I}\right)$ restringen uniformemente en $x$ $\mathrm{r}=1, \ldots, m$ y los $\mathrm{H} r$ son los anteriormente definidos

\section{Teorema}

Si las condiciones I-IV son satisfechas, entonces cuando $t \rightarrow 0$ la fórmula asintótica

$$
F(x, t) \sim F_{m}(x, t)=\Phi(x)+\varphi(x) \Omega(x) \sum_{k=1}^{m} H_{k}(x) t^{k}
$$

es válida para $F(x, t)$, en la que las $\mathrm{H} k$ satisfacen las condiciones del lema y $\mathrm{r} m(x, t)=\mathrm{F}(x, t)-\mathrm{F} m(x, t)=\mathrm{O}\left(\mathrm{t}^{\mathrm{m}+1}\right)$ uniformemente en todo el eje $O x$.

La teoría de las transformaciones asintóticas Pearson puede ser construida por analogía con las trasformaciones asintóticas normales.

Como se dijo anteriormente, sea la función $F(x, t)$ que satisface las condiciones I-IV, anteriormente mencionadas, y sea $\Phi(x)$ que satisface la condición

$$
\frac{\varphi^{\prime}}{\varphi}=-c_{0}(x) \equiv \frac{\omega(x)}{\Omega(x)} \equiv \frac{a_{1} x+a_{0}}{b_{2} x^{2}+2 b_{1} x+b_{0}}
$$

Si la variable aleatoria $\xi$ tiene función de distribución $F(x, t)$, entonces se puede decir que la variable aleatoria $\eta=\Phi^{-1}[F(\xi, t)] \equiv \mathrm{y}(\xi)$ está sujeta a la distribución con función de distribución $\Phi(x)$.

Si, además, la función $\tilde{y}$ es, en algún sentido, próxima a la función $y$, entonces, cabe suponer que la distribución de la variable aleatoria $\tilde{\eta}=\tilde{y}(\xi)$ será próxima a la distribución de $\eta$.
Para garantizar que la función $\Phi(x)$ se aproxima a la función de distribución $\tilde{y}(\xi)$ mejor que $F(x, t)$ se añade la siguiente condición:

V. $\mathrm{Si}\left(\mathrm{A}_{\mathrm{m}}(t), \mathrm{B} m(t)\right)$ es es el intervalo máximo y contiene el punto 0 , en el cual la función

$y_{m}(x)=x+\Omega(x) \sum_{k=1}^{m} P_{k}(x) t^{k}$

( $\mathrm{P}_{\mathrm{k}}, \Omega$ polinomios)

crece monótonamente, entonces cuando $\mathrm{t} \rightarrow 0$

$F\left[\mathrm{~B}_{\mathrm{m}}(t), t\right]-F\left[A_{m}(t), t\right]=1+O\left(t^{m+1}\right)$ ya que los coeficientes son polinomios, se puede hallar $\mathrm{C}>0$ y r $>0$ para los cuales $\forall|x| \geq 1$ la desigualdad

$\left|y_{m}^{\prime}(x)-1\right| \leq t(|x| / C)^{r}$ sea cumplida.

Así, la función $\mathrm{y}_{\mathrm{m}}(x)$ crece monótonamente en el intervalo $|\mathrm{x}|<\mathrm{Ct}^{-1 / \mathrm{r}}$

Esto es, se debe cumplir, para que la condición $\mathrm{V}$ sea válida, lo que la siguiente afirmación encierra:

Cuando $\mathrm{t} \rightarrow 0$

$P\left\{|\xi|<C t^{-1 / r}\right\}=F\left[C t^{-1 / \mathrm{r}}, \mathrm{t}\right]-F\left[-C t^{-1 / \mathrm{r}}, \mathrm{t}\right]=1+O\left(\mathrm{t}^{\mathrm{m}+1}\right)$

Ahora sí, se enuncia lo siguiente.

\section{Teorema}

Si se satisfacen las condiciones I-V, entonces, cuando $t \rightarrow 0$, la función de distribución $\mathrm{F} \eta(z, t)$ de la variable aleatoria $\eta=\mathrm{y}_{\mathrm{m}}(\xi)$ difiere de $\Phi(z)$ en $O\left(\mathrm{t}^{\mathrm{m}+1}\right)$, uniformemente, en cualquier segmento fijo $a \leq z \leq b, A<\mathrm{a}<\mathrm{b}<\mathrm{B}$.

Una amplia clase de transformaciones usuales de la variable aleatoria son llamadas transformaciones Pearson asintóticas regulares y para su descripción necesitamos el siguiente:

\section{Teorema}

Sea $\mathrm{U}_{m}$ un conjunto de funciones $\mathrm{u}(x, t)$ definidas en la región $|\mathrm{x}|<\infty,<t \leq \mathrm{T}$, para cada una de las cuales

1) existe la derivada parcial $\partial^{\mathrm{m}+1} / \partial \mathrm{t}^{\mathrm{m}+1}$ la cual es continua en el eje de las abscisas

2) $\mathrm{F}[B(t), t]-\mathrm{F}[A(t), t]=1+\mathrm{O}\left(\mathrm{t}^{m+1}\right)$ cuando $\mathrm{t} \rightarrow 0$ donde $A(t)<x<B(t)$ es el máximo intervalo que contiene el punto en el cual la función $u(x, t)$ crece monótonamente.

Si las condiciones I-V son satisfechas y $u(x, t) \in \mathrm{U}_{m}$, entonces, cuando, $\mathrm{t} \rightarrow 0$ la función de distribución $\mathrm{F} \eta(z, t)$ de la variable aleatoria $\eta=\mathrm{u}(\xi, t)$ satisface la relación

$F \eta(z, t)=\Phi(z)+O\left(\mathrm{t}^{m+1}\right)$

Si y sólo si

$\mathrm{u}(x, t)=y_{\mathrm{m}}(x)+O\left(\mathrm{t}^{m+1}\right)$

Donde

$y_{m}(x)=x+\Omega(x) \sum_{k=1}^{m} P_{k}(x) t^{k}$

Los restos de las fórmulas precedentes son uniformes en todo segmento fijo.

\section{Fórmula asintótica para la distribución Beta $B$}

Con la notación de Pearson, la distribución B está dada por la fórmula: $0 \leq x \leq 1$ 


$$
I_{x}(p, q)=\frac{1}{\mathrm{~B}(p, q)} \int_{n}^{x} u^{p-1}(1-u)^{q-1} d u
$$

Se sabe que: $\mathrm{B}(p, q)=\int_{0}^{1} u^{p-1}(1-u)^{q-1} d u$

y son parámetros de la distribución

Si $\mathrm{p}=$ const $\quad$ y $\quad q \rightarrow \infty$ transformamos

$\mathrm{I} x(p, q)=I(x q, p)+O(1 / q)$

Donde

$$
I(y, p)=\frac{1}{\Gamma(p)} \int_{0}^{y} v^{p-1} e^{-v} d v
$$

y

$$
\Gamma(p)=\int_{0}^{\infty} v^{p-1} e^{-v} d v
$$

Se hace $t=1 / q F(x, t)=I_{x t}(p, 1 / t)$ y $\Phi(x)=I(x, p)$ donde $\mathrm{x}>0(\mathrm{~A}=0, B=\infty)$ considerando

$$
\frac{\varphi^{\prime}}{\varphi}=-c_{0}(x) \equiv \frac{\omega(x)}{\Omega(x)} \equiv \frac{a_{1} x+a_{0}}{b_{2} x^{2}+2 b_{1} x+b_{0}}
$$

$\mathrm{y} \quad(y, p)=\frac{1}{\Gamma(p)} \int_{0}^{y} v^{p-1} e^{-v} d v$

Siendo: $\Omega(x) \equiv x$

Es fácil mostrar, para $0<x<1 / t$

$\frac{f^{\prime}(x, t)}{f(x, t)}=\frac{p-1-x}{x}+t \frac{1-x}{1-x t}=\frac{\varphi^{\prime}}{\varphi}+\sum_{k=1}^{m} x^{k-1}(1-x) t^{k}+t^{m+1} \frac{x^{m}(1-x)}{1-x t}$

Para todo $t$, empezando por alguno $\mid\left(x^{m}(1-x) /(1-x t) \mid \leq 2 \mathrm{x}^{m}\right.$ $|1-x|$ en el segmento $\mathrm{a}_{\mathrm{t}} \leq x \leq \mathrm{b} t$ en el cual

$a_{t}=0 \quad b_{t}=-\log t / t^{\varepsilon}$ y $0<\varepsilon<1 / 2$

Es fácil ver que son satisfechas las condiciones I, II y III. Para un suficientemente pequeño

$$
\begin{aligned}
& F\left[a_{t}, t\right]-F\left[b_{t}, t\right] \geq 1-\frac{4}{\Gamma(\mathrm{p}+1)} t^{t^{-\varepsilon}-p} \geq 1-t^{m+1} \\
& \Phi\left(b_{t}\right)-\Phi\left(a_{t}\right) \sim 1-\frac{1}{\Gamma(p)}\left(-\frac{\log t}{t^{\varepsilon}}\right)^{p-1} t^{t^{-\varepsilon}} \geq 1-t^{m+1}
\end{aligned}
$$

De acuerdo con los teoremas enunciados se obtiene:

$$
F(x, t)=\Phi(x)+\varphi(x) x \frac{x+p-1}{2} t+O\left(t^{2}\right)
$$

Uniformemente en el eje de las abscisas y

$$
y(x)=x+x \frac{x+p-1}{2} t+o\left(t^{2}\right)
$$

Uniformemente en cualquier segmento fijo $0<a \leq x \leq b$

Se halla la transformación $u(x, t)$ para cada $\mathrm{F} \eta-\Phi=O\left(t^{2}\right)$

Se asume $u$ como una función lineal fraccionaria de $x$ con coeficientes que dependen linealmente de $t$

Esta función toma la forma

$x[2+\mathrm{t}(\alpha+\mathrm{p}-1)] /[2+t(\alpha-x)]$

Donde $\alpha$ es un número arbitrario

Se hace

$\mathrm{s}=t /[2+t(\alpha+p-1)]$

Se tiene

$$
u(x, s)=\frac{x}{1-s(x+p-1)}
$$

Si la distribución de la variable aleatoria $\xi$ es dada por la función $F(x, t)$, para $\alpha=0$ la densidad de probabilidad $f \eta(x, s)$ de la variable aleatoria $\eta=u(\xi, s)$ tendrá la forma (quedando por introducir el factor de normalización).

$\mathrm{x}^{\mathrm{p}-1}(1-s x)^{(1 / \mathrm{s}-\mathrm{p}-1) / 2}(1+s x)^{-(1 / \mathrm{s}+\mathrm{p}+1) / 2}$

Donde $0<x<1 / s$

Es decir, $f \eta(x, s)$ es siempre una función respecto a $s$

La distribución de $\eta$ satisface las condiciones I-IV. Además la expansión

$$
c(x, t)=\sum_{k=0}^{m} c_{k}(x) t^{k}+t^{m+1} R_{m}(x, t)
$$

Se realiza en potencias de $s^{2}=(2 q+p-1)^{-2}$

Por ejemplo, cuando $s \rightarrow 0$

$$
\begin{gathered}
F_{n}(x, s)=\Phi(x)+\varphi(x) x \frac{2 x^{2}-(p-1)(x+p+1)}{6} s^{2}+O\left(s^{4}\right) \\
y_{\eta}(x)=x+x \frac{2 x^{2}-(p-1)(x+p+1)}{6} s^{2}+O\left(s^{4}\right)
\end{gathered}
$$

Otra transformación posible es:

$$
\eta=-\frac{2 q+p-1}{2} \log \left(1-\frac{\xi}{q}\right)=-\frac{1}{2 s} \log \left[1-\frac{2 s \xi}{1-s(p-1)}\right]
$$

Con

$$
F_{n}(x, s)=\Phi(x)-\varphi(x) x \frac{(p-1)(x+p+1)}{6} s^{2}+O\left(s^{4}\right)
$$

De acuerdo con teoremas precedentes, cuando $\mathrm{s} \rightarrow 0$

$$
x(y)=\frac{y}{1+\left[2 y^{2}-(p-1) y-\left(p^{2}-1\right)\right] \frac{s^{2}}{6}}+O\left(s^{4}\right)
$$

Uniformemente en cualquier intervalo finito de variación de $y$.

Es decir, si tiene la distribución

$$
I(\gamma, p)=\frac{1}{\Gamma(p)} \int_{0}^{\gamma} v^{p-1} e^{-v} d v
$$

Entonces $\mathrm{x}(\gamma)$ está distribuida como $(2 q+p-1) \beta /(2-\beta)$ donde $\beta$ tiene distribución

$$
I_{\beta}(p, q)=\frac{1}{\mathrm{~B}(p, q)} \int_{0}^{\beta} u^{p-1}(1-u)^{q-1} d u
$$

En consecuencia, la variable aleatoria

$$
\beta^{*}=\frac{2 \gamma}{\frac{1}{s}+\gamma-\left[\left(p^{2}-1\right)+(p-1) \gamma-2 \gamma^{2}\right] \frac{s}{6}}
$$

Aproxima bien la distribución $\mathrm{B}$ con parámetros $p$ y $q$ donde si $q \rightarrow 0$, entonces

$\mathrm{P}\left\{\beta^{*}<x\right\}=\mathrm{I}_{x}(p, q)+\mathrm{O}\left(s^{5}\right)$

\section{Resultados y discusión}

Método Monte Carlo. Cálculo de la función de distribución con densidad Beta

Se quiere calcular

$I=\int_{-\infty}^{t} f(x) d x$

Donde $f$ es una densidad correspondiente a una distribución beta o Pearson tipo I. 
$f(x)=\frac{\alpha^{2 m} \beta^{2 n}}{(\alpha+\beta)^{m+n+1} \mathrm{~B}(m+1, n+1)}(\alpha+x)^{m}(\beta-x)^{n}$

Donde:

$x \in[-\alpha, \beta] \alpha, \beta>0$

y $\quad \alpha=0$

$\beta=1$

$f(x)=\frac{\Gamma(m+n)}{\Gamma(m) \Gamma(n)}(x)^{m-1}(1-x)^{n-1}$

Se puede calcular la integral

$$
\begin{gathered}
I=\int_{0}^{t} f(x) d x \\
I=\int_{0}^{t} \frac{\Gamma(m+n)}{\Gamma(m) \Gamma(n)}(x)^{m-1}(1-x)^{n-1} d x
\end{gathered}
$$

Para $\mathrm{t}=0,6$ y $\mathrm{m}=3, \mathrm{n}=4$

$$
\begin{gathered}
I=\int_{0}^{0.6} \frac{\Gamma(m+n)}{\Gamma(m) \Gamma(n)}(x)^{m-1}(1-x)^{n-1} d x \\
I=\int_{0}^{0.6} \frac{\Gamma(7)}{\Gamma(3) \Gamma(4)}(x)^{2}(1-x)^{3} d x
\end{gathered}
$$

Tabla 1. Cálculo función de la distribución Beta

\begin{tabular}{|c|c|c|c|}
\hline $\mathbf{i}$ & $t_{i}$ & $x_{i}$ & $f\left(x_{i}\right)$ \\
\hline $\mathbf{1}$ & 0,8574 & 0,8574 & 0,12790184 \\
\hline $\mathbf{2}$ & 0,4575 & 0,4575 & 2,00508448 \\
\hline $\mathbf{3}$ & 0,4999 & 0,4999 & 1,87537485 \\
\hline $\mathbf{4}$ & 0,7627 & 0,7627 & 0,46639293 \\
\hline $\mathbf{5}$ & 0,4315 & 0,4315 & 2,05259968 \\
\hline $\mathbf{6}$ & 0,6987 & 0,6987 & 0,80118006 \\
\hline $\mathbf{7}$ & 0,0387 & 0,0387 & 0,07982704 \\
\hline $\mathbf{8}$ & 0,5581 & 0,5581 & 1,61267666 \\
\hline $\mathbf{9}$ & 0,6531 & 0,6531 & 1,06837451 \\
\hline $\mathbf{1 0}$ & 0,5735 & 0,5735 & 1,53100004 \\
\hline $\mathbf{1 1}$ & 0,6092 & 0,6092 & 1,32902976 \\
\hline $\mathbf{1 2}$ & 0,1791 & 0,1791 & 1,06466552 \\
\hline $\mathbf{1 3}$ & 0,9746 & 0,9746 & 0,00093391 \\
\hline $\mathbf{1 4}$ & 0,0118 & 0,0118 & 0,00806213 \\
\hline $\mathbf{1 5}$ & 0,0986 & 0,0986 & 0,42722607 \\
\hline $\mathbf{1 6}$ & 0,8057 & 0,8057 & 0,28570404 \\
\hline $\mathbf{1 7}$ & 0,5161 & 0,5161 & 1,810864 \\
\hline $\mathbf{1 8}$ & 0,2961 & 0,2961 & 1,83468268 \\
\hline $\mathbf{1 9}$ & 0,1494 & 0,1494 & 0,82419235 \\
\hline $\mathbf{2 0}$ & 0,8153 & 0,8153 & 0,25129672 \\
\hline $\mathbf{2 1}$ & 0,7003 & 0,7003 & 0,79209949 \\
\hline $\mathbf{2 2}$ & 0,6928 & 0,6928 & 0,83489266 \\
\hline $\mathbf{2 3}$ & 0,6961 & 0,6961 & 0,81599335 \\
\hline $\mathbf{2 4}$ & 0,203 & 0,203 & 1,25175199 \\
\hline $\mathbf{2 5}$ & 0,3503 & 0,3503 & 2,01915717 \\
\hline $\mathbf{2 6}$ & 0,9005 & 0,9005 & 0,04792785 \\
\hline & & & \\
\hline
\end{tabular}

\begin{tabular}{|l|l|l|l|}
\hline $\mathbf{2 7}$ & 0,4518 & 0,4518 & 2,01771953 \\
\hline $\mathbf{2 8}$ & 0,3186 & 0,3186 & 1,92685565 \\
\hline $\mathbf{2 9}$ & 0,7982 & 0,7982 & 0,31415081 \\
\hline $\mathbf{3 0}$ & 0,1114 & 0,1114 & 0,52244498 \\
\hline
\end{tabular}

De la Tabla 1:

$$
I=\frac{b-a}{N} \cdot \sum_{i=1}^{N} f\left(x_{i}\right)=\frac{0,6}{30} \cdot(30,0000627)=0,60000125
$$

\section{Aproximación Asintótica}

\section{Cálculo de la función de distribución con densidad Beta}

$$
I_{x}(p, q)=\frac{1}{\mathrm{~B}(p, q)} \int_{0}^{x} u^{p-1}(1-u)^{q-1} d u
$$

El valor exacto para

$$
x=0,6 \quad p=3 \text { y } q=4
$$

$$
\begin{gathered}
I=\int_{0}^{0.6} \frac{\Gamma(7)}{\Gamma(3) \Gamma(4)}(x)^{2}(1-x)^{3} d x \\
I=0,54432
\end{gathered}
$$

Siendo: un infinitésimo de quinto orden, se desprecia en la aproximación asintótica.

\section{Conclusiones}

El método Monte Carlo es comparativamente más ágil que otros métodos de aproximación que exigen una mayor elaboración matemática. Puede ser aplicado a las distribuciones de Pearson, llevando a resultados satisfactorios y evitando el proceso de la convergencia asintótica, detallado en el presente documento.

Como se ha visto, el método de Monte Carlo es un recurso para calcular integrales definidas que difícilmente pueden ser evaluadas por métodos directos.

\section{Literatura citada}

Metropolis, N. y Ulam, S. 1949. The Monte Carlo method. J. Amer. statistical assoc., 44(247): 335-341.

Todos los siguientes no se mencionan en el texto Montel, Paul "Series de polinomios" Gauthier Villars 1928.

Hardy, G.H. “Series Divergentes” Oxford editorial 1946.

Carlson, B.C. 1983. "Funciones especiales de la matemática aplicada"Academic Press.

Luke, Y. 1949. "Integrales de las funciones de Bessel" Mc Graw-Hill.

Cramer, H "Métodos matemáticos de la estadística".

Korokiuk, V.1986."Manual de la Teoría de Probabilidades y la Estadística Matemática” Ed. Mir 1986. 